\title{
La actuación de agentes indígenas de salud en la región de Alto Rio Negro, Brasil: relaciones entre autoatención y biomedicina*
}

\author{
ANA LÚCIA PONTES, SERGIO REGO Y LUIZA GARNELO
}

Se analiza la actuación de los agentes indígenas de salud en el Alto Rio Negro para reflexionar sobre la implementación del subsistema de atención a la salud indígena en Brasil. Destacan las relaciones de los agentes con las formas de atención utilizadas por las familias para resolver sus problemas de salud. La base teórica es el análisis de los modelos de atención de Eduardo Menéndez desde una perspectiva antropológica. Se anota que las prácticas de autoatención son la primera instancia de resolución de los problemas de salud y que los medicamentos son centrales en las relaciones transaccionales entre autoatención y biomedicina. La intervención de los agentes facilita el acceso a los servicios biomédicos, es decir, funcionan como generadores de prácticas de autoatención.

PALABRAS CLAVE: autoatención, salud de los pueblos indígenas, agentes indígenas de salud, pluralismo médico, Amazonia

ANA LÚCIA PONTES

Escola Nacional de Saúde Pública Sérgio Arouca, Fundación Oswaldo Cruz, Brasil analupontes64@gmail.com

SERGIO REGO

Conselho Nacional de Desenvolvimento Científico e Tecnológico, Escola Nacional de Saúde Pública Sérgio Arouca, Fundação

Oswaldo Cruz, Río de Janeiro, Brasil starego@gmail.com

LUIZA GARNELO

Conselho Nacional de Desenvolvimento Científico e Tecnológico, Instituto Leônidas \& Maria Deane, Fundação Oswaldo Cruz, Manaos, Amazonas, Brasil malupereira2011@gmail.com

Traducción: Sandra Carolina Portela García y Juana Valentina Nieto Moreno
The Performance of Indigenous Health Agents in the Upper Rio Negro Region, Brazil: Relationships between Self-Care and Biomedicine

This article analyzes the performance of the indigenous health agents in the Alto Rio Negro region, with the objective of reflecting on the implementation of the indigenous health care in Brazil. It highlights the relationships these agents establish with the forms of attention used by families to solve their health problems. Theoretically, it is based on the contributions of Eduardo Menéndez on the analysis of care models from an anthropological perspective. It was noted that self-care practices were the first instance of resolution of health problems and that medications are central to the transactional relationships between self-care and biomedicine. The actions of indigenous health agents facilitate access to biomedical services, functioning as well as generators of self-care practices.

KEYWORDS: self-care, health of indigenous peoples, indigenous health agents, medical plurality, Amazonas 


\section{Introducción}

$\mathrm{n}$ este artículo discutiremos el trabajo de los agentes indígenas de salud (AIS) en dos comunidades indígenas de la región del Alto Rio Negro, Amazonas. Los agentes viven en las comunidades indígenas y son contratados por el Subsistema de Atención a la Salud Indígena (SASI) brasileño para atender a estas poblaciones.

En Brasil, los pueblos indígenas ${ }^{1}$ presentan los peores indicadores de salud, con tasas altas de mortalidad materno-infantil, niveles elevados de desnutrición y enfermedades infecto-parasitarias, a lo que se suma el incremento de enfermedades crónicas y no transmisibles, números alarmantes de suicidio, abuso de sustancias y muertes por violencia (Basta, Orellana y Arantes, 2012). Hasta la década de 1980, la perspectiva de que estos pueblos se extinguirían justificó respuestas puntuales y esporádicas para esta situación.

Sin embargo, durante el proceso de redemocratización, a finales de la década de 1980 se consolidaron nuevos derechos constitucionales para los pueblos indígenas y su lucha por una nueva política nacional de atención a la salud se fortaleció. En 1999 se aprobó la Ley Arouca (Presidência da República, 1999), que decretó la creación del sasi dentro del Sistema Único de Salud (Presidência da República, 1990). En el marco de esta ley, las poblaciones indígenas empezaron a tener acceso a las acciones de salud con la organización de 34 Distritos Sanitarios Especiales Indígenas (DSEI) en sus territorios.

* Apoyo financiero del Programa Pesquisa Estratégica em Saúde (PAPES VII), de la Fundação Oswaldo Cruz, y del Conselho Nacional de Desenvolvimento Científico e Tecnológico (cNPq). Agradecemos la traducción al español de Sandra Carolina Portela García y Juana Valentina Nieto.

1 La población indígena brasileña estimada por el censo de 2010 es de aproximadamente 817900 personas, pertenecientes a 305 grupos étnicos, hablantes de 274 lenguas, de las cuales 63.8\% reside en áreas rurales (IBGE, 2012). 
El modelo de atención del Subsistema se detalla en la Política nacional de atención a la salud de los pueblos indígenas (PNASPI; Fundação Nacional de Saúde, 2002), en la que se describe la organización de una red de servicios de atención primaria en territorios indígenas. Este modelo de atención en las comunidades se basa en la actuación de los AIS, apoyados por un equipo multidisciplinario, y tiene como directriz la noción de "atención diferenciada", que se refiere al reconocimiento y el respeto a la diversidad sociocultural de los pueblos indígenas, principalmente a sus conocimientos y prácticas en salud (Ferreira, 2015; Langdon, 2004).

Varios autores han discutido las limitaciones de la implantación del sAsI. Garnelo (2012) señala que no ha logrado mejorar de manera significativa la situación de la salud de las poblaciones indígenas, dado que la atención es discontinua y de poca efectividad debido a las precariedades en su implementación y la alta rotación de los profesionales. Cardoso (2015) destaca que la organización de la atención reproduce las características del modelo biomédico hegemónico, en la ejecución de los procedimientos, el consumo de medicamentos y la estandarización de las acciones. Ferreira (2015) demuestra que la ausencia de claridad en la dirección de la atención diferenciada ha llevado a una noción esencializada de la cultura, que no considera las asimetrías entre medicinas indígenas y sistema oficial. De esta forma, las dimensiones culturales aún se entienden como barreras, lo que perpetúa una perspectiva integracionista y colonizadora de las medicinas tradicionales (Ferreira, 2015).

En este escenario, nuestra investigación pretende profundizar en la comprensión de la implementación de este modelo de atención diferenciada a partir de la actuación de los AIs. Esta elección se justifica porque debido a la alta rotación de los profesionales no indígenas, en muchos DSEI y comunidades sólo los agentes trabajan de forma regular, sobre todo en las regiones más alejadas (Langdon, Diehl y Dias-Scopel, 2014). Además, los objetivos de la atención diferenciada se basan en gran medida en la acción de los AIS porque se entiende que, al ser habitantes de las comunidades indígenas, deberían mediar la relación entre los servicios de salud y las prácticas locales (Langdon, Diehl y Dias-Scopel, 2014; Erthal, 2003; Novo, 2009).

Nuestro análisis del modelo de atención se enfocará en su dimensión operativa, o sea, en las relaciones entre los sujetos de las prácticas de salud y las tecnologías utilizadas para resolver los problemas y necesidades de salud (Teixeira y Solla, 2006). Así, desarrollamos una investigación etnográfica a partir de las rutinas y prácticas de los AIs para entender las relaciones entre las formas de atención utilizadas por las familias indígenas en la búsqueda de una solución a sus problemas de salud.

Para reflexionar sobre las normas de la atención diferenciada, buscamos un referente teórico que permitiera considerar la diversidad de prácticas y saberes de salud existentes en los territorios indígenas. La perspectiva antropológica sobre modelos de atención propuesta por Menéndez (2003; 2009) nos pareció adecuada para reconocer y valorar el pluralismo médico.

\section{La perspectiva antropológica sobre saberes, formas y modelos de atención}

La construcción de nuestro referente teórico se basó en la propuesta antropológica de Menéndez (2009) sobre saberes, formas y modelos ${ }^{2}$ de atención para

2

Los términos adoptados son construcciones de Menéndez (2009). Los saberes se refieren a las representaciones y prácticas de los sujetos y curadores profesionales; las formas se entienden como las trayectorias y experiencias individuales; los modelos son construcciones metodológicas para identificar de manera provisional características y funciones. 
analizar los procesos de salud/enfermedad/atención en dos comunidades indígenas en la región del Alto Rio Negro. Este enfoque se justifica en la medida en que la discusión sobre el pluralismo médico aún no permea los debates sobre modelos de atención y políticas de salud en Brasil.

En el contexto brasileño, los debates sobre modelos de atención se concentran en la discusión sobre el desarrollo y la crítica de la medicina científica, y la construcción de modelos alternativos para los sistemas de salud, como la Estrategia de Salud de la Familia (Silva Junior, 2006; Teixeira y Solla, 2006). Estas investigaciones a menudo se enfocan en los saberes y prácticas de los profesionales y gestores de salud, sin articular necesariamente la experiencia y perspectiva de los sujetos y grupos sociales. En estos análisis no se considera la noción de que los sujetos y grupos sociales hacen uso de una o más formas de atención para solucionar sus problemas de salud — la idea de pluralismo médico-.

En el marco de la PNASPI identificamos los mismos desafíos, en la medida en que se discute el modelo de atención del Subsistema como sinónimo de acceso creciente a servicios de salud. Desde la lógica del SASI, la atención empieza gracias a la actuación de los AIS en los puestos de la salud localizados en las comunidades indígenas, con el apoyo de otros profesionales de la salud - médicos, enfermeros, técnicos de enfermería, odontólogos y técnicos de salud bucal一, quienes permanecen en las unidades de salud de referencia regional denominadas Polo Base. La continuidad del cuidado médico tiene lugar en los centros urbanos, en las Casas de Salud del Indio (Casai), en las que se proveen los servicios de salud de mayor complejidad.

El foco del modelo de atención, según el SASI, está en la oferta de servicios de salud, pero nuestra intención en este trabajo es traer al debate el análisis del conjunto de saberes y formas de atención utilizado por los indígenas, así como sus procesos relacionales y transaccionales, principalmente con la biomedicina.
A pesar de la hegemonía de la biomedicina en los servicios y sistemas de salud, la autoatención es el recurso más utilizado por los sujetos para la mayoría de sus problemas de salud (Menéndez, 2009). La autoatención es un proceso estructural en la vida de los grupos sociales y se refiere a las representaciones y prácticas relacionadas con los procesos de salud/ enfermedad/atención desarrolladas con autonomía, o autonomía relativa, de los especialistas o profesionales (Menéndez, 2009). La autoatención involucra la elaboración de diagnósticos, la evaluación de riesgos, el análisis del pronóstico de las enfermedades y la decisión de buscar curadores o especialistas profesionales. Una vez incluidos los curadores profesionales en el proceso, la autoatención continúa en la medida en que los sujetos deciden seguir de manera parcial o total los tratamientos propuestos, buscar otros especialistas o abandonar a los curadores profesionales. Menéndez destaca: "la autoatención no debe ser pensada como un acto que los sujetos y grupos desarrollan aislada y autónomamente, sino como un proceso transaccional entre éstos y las diferentes formas de atención que operan como sus referentes" (2009: 57).

Además, a partir de la autoatención, los sujetos y grupos sociales establecen entre las formas de atención otras complementariedades, yuxtaposiciones y articulaciones. ${ }^{3}$ Estas articulaciones dependen de las condiciones socioeconómicas y culturales, pero se facilitan tanto por el pragmatismo y la urgencia en la resolución de los problemas de salud de los sujetos, como por el hecho de que los indicadores diagnósticos son comunes a las diferentes formas de atención (Menéndez, 2009).

En términos metodológicos, la mejor estrategia para reconocer la diversidad de saberes y formas

Menéndez (2009: 31) identifica cinco formas principales de atención: a) el tipo biomédico; b) el tipo popular o tradicional; c) las alternativas, paralelas o new age; d) las diferentes tradiciones médico-académicas, y e) la autoatención. 
de atención y sus articulaciones sería describir lo que hacen, dicen y usan los sujetos y grupos sociales, y no partir de los especialistas y profesionales. Menéndez (2009) sugiere que se incluya a todos los sujetos participantes en contextos particulares y todas las representaciones y prácticas relacionadas con el proceso salud/enfermedad/atención.

Las articulaciones entre las formas de atención también ocurren a partir de los especialistas y profesionales, sin embargo, Menéndez (2009) apunta que su tendencia es actuar garantizando su propia hegemonía. De esta forma, la biomedicina es la forma de atención que más crece, incluso por la apropiación de otras formas de atención. Como formas de expansión de la biomedicina destaca la biologización de los problemas de salud y la medicalización, y la atención primaria ejerce un importante papel en ese proceso. Cabe resaltar que en la actualidad la biomedicina es una fuente, directa o indirecta, de saberes y prácticas involucradas en la autoatención (Menéndez, 2009).

Al reflexionar sobre estas cuestiones en el contexto de los pueblos indígenas, Fóller (2004) caracteriza como intermedicalidad el espacio de encuentro entre biomedicina y medicina tradicional. ${ }^{4}$ Ese encuentro formaría parte del proyecto colonial en los territorios indígenas e involucraría desigualdades y conflictos. Sin embargo, debemos considerar que en ese proceso los pueblos indígenas poseen agencia social y producen nuevos conocimientos y prácticas.

\section{Contexto local del estudio}

\section{LA REGIÓN DE ALTO RIO NEGRO Y LA HISTORIA DEL CONTACTO}

Esta investigación se llevó a cabo en la región del DSEI Alto Rio Negro, que se ubica en el Noroeste amazónico y abarca tierras indígenas en los municipios de São Gabriel da Cachoeira, Barcelos y
Santa Isabel do Rio Negro. Según datos del Sistema de Información de Atención a la Salud Indígena (SIASI, 2013), en esa región viven cerca de 36390 personas, identificadas como pertenecientes a 45 etnias. Las principales son, de mayor cantidad poblacional a menor, baré, baniwa, tukano, hupde, tariano, desano, curipako, piratapuia y warekena. La región se divide en términos geopolíticos por los principales cursos de los ríos y sus afluentes (véase el mapa 1). Este territorio posee una importante diversidad sociocultural y lingüística.

La historia de contacto y colonización en la región se remonta al siglo XVIII, con la llegada de los misioneros que capturaron y mataron una gran cantidad de indígenas (ISA y FOIRN, 2006). A partir de 1850 , se institucionalizó un programa de "civilización y catequesis" (Andrello, 2006) que buscaba atraer y convencer a los indígenas de desplazarse a los poblados y aldeas coloniales en las márgenes del río para someterlos a un régimen de trabajo obligatorio en la extracción de recursos naturales (ISA y FOIRN, 2006).

Desde finales del siglo XIX hasta mediados del xx, llega a la región el auge de la economía del caucho, que aumentó la demanda de trabajo indígena y condujo a una gran cantidad de familias al desplazamiento estacional (Andrello, 2006; ISA y FOIRN, 2006). Este periodo se caracterizó por la explotación, el desplazamiento forzado y la violencia contra los pueblos indígenas de la región. Las actividades de los misioneros se retomaron en 1880, también con un sistema de trabajo obligatorio en la construcción y el extractivismo. En 1915 llegaron los salesianos, que controlaron los abusos de los

4

En este trabajo utilizaremos la denominación de medicamentos y medicina tradicional, pues es una categoría emic en el contexto de Rio Negro. En términos de debate, estamos de acuerdo con la discusión de Ferreira (2013) y Menéndez (1987). 


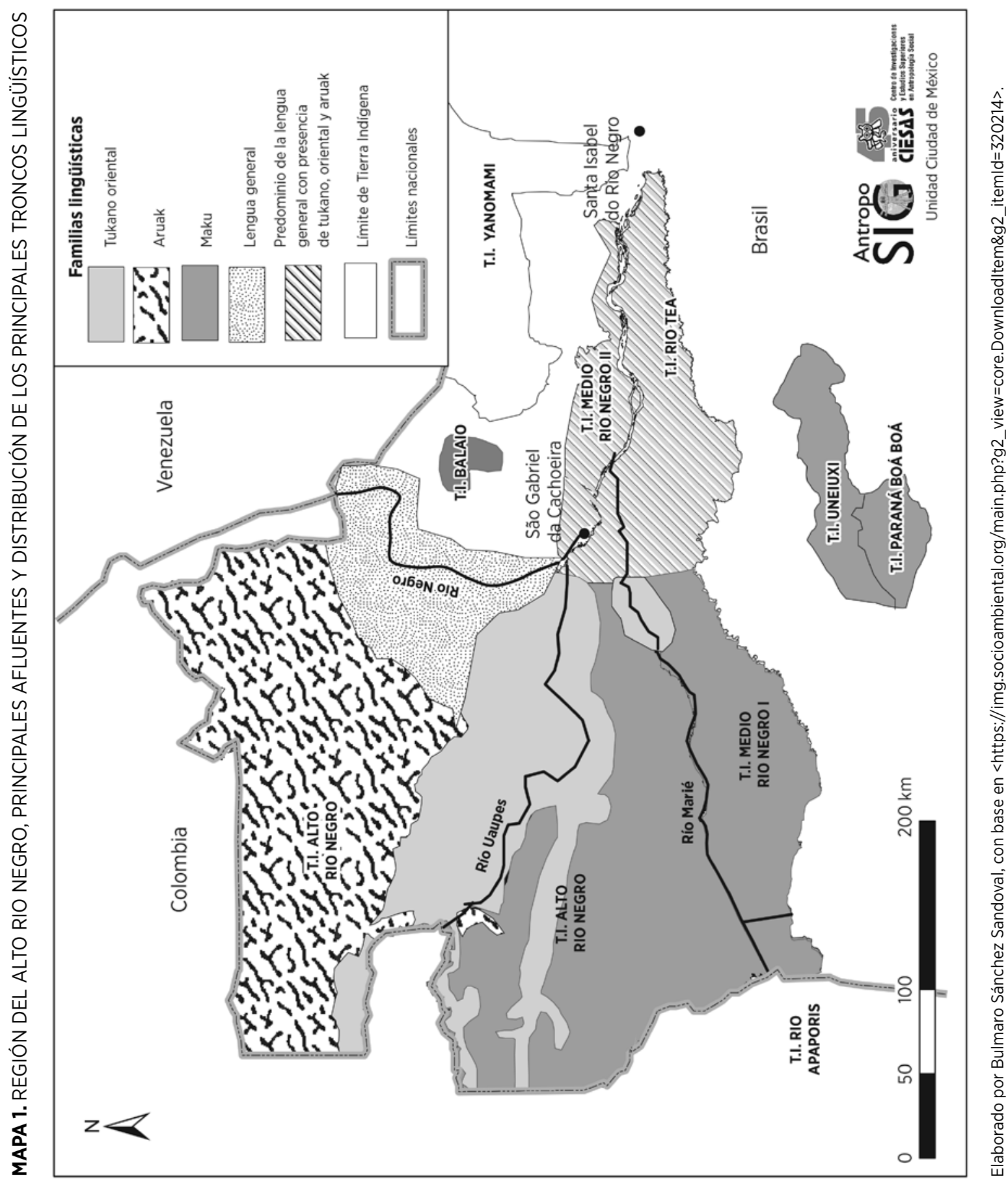


comerciantes, construyeron misiones e interfirieron en la vida de los indígenas (Wright, 1992).

A partir de la década de 1970 empezó la organización del movimiento indígena en la región, con el objetivo principal de la reivindicación de sus territorios. Durante el final de la década de 1980 y principios de la siguiente, ocurrió un intenso proceso de negociación de los líderes indígenas con las autoridades gubernamentales. En este proceso de articulación y movilización política de los movimientos indígenas se creó la Federación de las Organizaciones Indígenas del Alto Rio Negro (FOIRN), en abril de 1987. Los líderes consiguieron la demarcación del Alto Rio Negro entre diciembre de 1995 y mayo de 1996. Desde entonces fortalecieron su actuación en otros frentes, como el de las políticas de educación, desarrollo sostenible y salud (ISA y FOIRN, 2006).

\section{ASPECTOS GENERALES DE LA ORGANIZACIÓN SOCIOCULTURAL DE LOS BANIWA}

Nuestra investigación se realizó en dos comunidades de la etnia baniwa, cuya lengua pertenece a la familia lingüística aruak (véase el mapa 1). En la actualidad, la etnia baniwa posee una población estimada en 6424 personas, distribuidas a lo largo del Rio Içana y sus afluentes (SIASI, 2013). El Alto Içana se reconoce como área del pueblo Coripaco, un grupo de mayoría evangélica que sufre la influencia cotidiana del Ejército Nacional de Colombia, por estar ubicado en la frontera con ese país (Garnelo, 2003). Por encima de la aldea Tunuí se localiza la región del Medio Içana, ocupada por los baniwa, hablantes de su lengua. La mayoría de las comunidades de esta región son evangélicas y de pequeño porte, pese a que son el locus de mayor dinamismo del movimiento indígena en la región. El área del Bajo Içana se encuentra entre la desembocadura del Rio Içana y la aldea Tunuí, más cerca de la ciudad de São Gabriel da Cachoeira, por lo cual experimentó un proceso de colonización más contundente, con intensa actuación de misiones evangélicas, católicas y otras agencias de la sociedad nacional que acentuaron las transformaciones de estos grupos (Garnelo, 2003).

Desde el punto de vista de la historia de contacto con el blanco, los baniwa comparten una historia común con el resto de pueblos rionegrinos, con algunas singularidades, como la conversión colectiva a la religión evangélica y un milenarismo con eclosión periódica de "movilización interna contra el violento proceso colonizador" (2003: 20). La conversión evangélica en masa ocurrió a partir de 1948, con la llegada de la misionera estadounidense Sophia Müller. Este proceso de conversión instauró un conflicto entre católicos y evangélicos, y restringió, sobre todo entre evangélicos, el mantenimiento de prácticas chamánicas, técnicas tradicionales de cura y rituales de pubertad masculinos (Garnelo, 2003). No obstante, este pueblo todavía preserva algunas de sus tradiciones y ha limitado la entrada del proceso civilizatorio en sus territorios (Garnelo y Wright, 2001).

La sociedad baniwa, como otros pueblos del Alto Rio Negro, se organiza en unidades de descendencia patrilineal y patrilocal (Garnelo y Wright, 2001). Este grupo se divide en cinco o seis grupos de parientes consanguíneos, denominados fratrías, que a su vez se subdividen en sibs. En esta forma de organización social, las fratrías mantienen un estatus igualitario entre sí, mientras que las relaciones entre y dentro de las sibs son jerarquizadas (Garnelo, 2003). También se observan relaciones de subordinación de género y generación. Estas jerarquías y relaciones de subordinación garantizan el orden político y optimizan el uso de los recursos naturales entre fratrías (Garnelo, 2003).

La organización social de la vida cotidiana y la reproducción social de los baniwa se basa en un sistema de saberes míticos (Garnelo y Wright, 2001). Según Garnelo y Buchillet (2006), las narrativas 


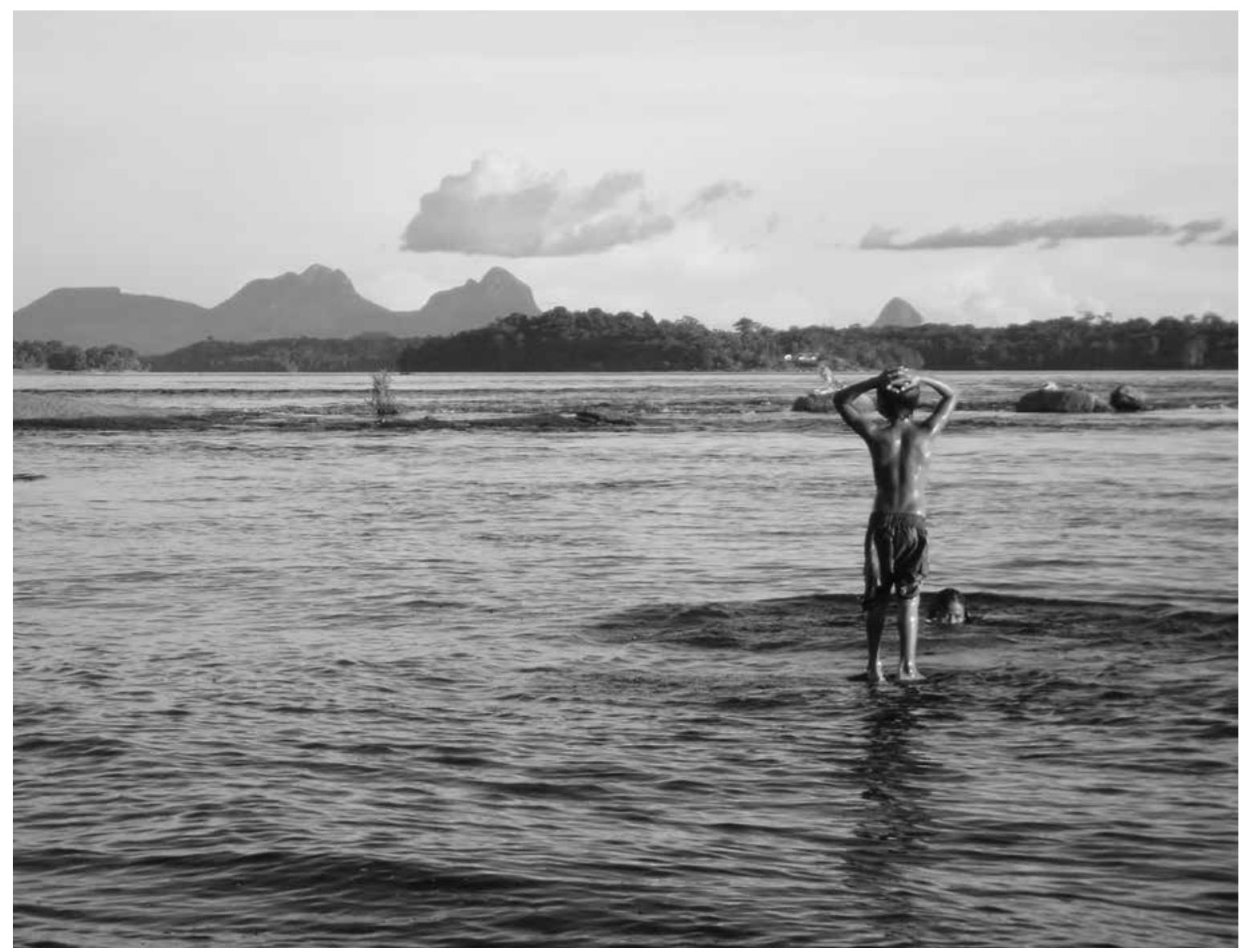

ANA LúciA Pontes • La bela adormecida, denominación coloquial de los cerros en São Gabriel da Cachoeira, enero de 2009.

míticas también son fundamentales para la comprensión del origen, mantenimiento y clasificación de las enfermedades en esta sociedad.

\section{Metodología}

Los datos discutidos en ese trabajo forman parte de la tesis de doctorado Atención diferenciada y trabajo del agente indígena de salud (Pontes, 2013). Además, Pontes y Garnelo cuentan con experiencia de trabajo previa en la región, en apoyo e impartición de cursos de formación técnica para los AIS. Esta experiencia las familiarizó con el contexto local en términos culturales, políticos y sociales, así como con cuestiones relacionadas con la organización y la actuación de los servicios de salud de los AIS.

La estrategia metodológica escogida fue la observación participante, pues se parte del supuesto de que "la interacción directa con las personas en su vida cotidiana puede ayudar a comprender mejor sus concepciones, prácticas, motivaciones, comportamientos y procedimientos, así como los significados que le atribuyen a sus prácticas" (Chizzotti, 2010: 65).

El trabajo de campo se llevó a cabo durante los meses de octubre y diciembre de 2011, en dos comunidades del Bajo Içana: Açaí, principalmente 
católica, y Buriti, evangélica. La etnia predominante era baniwa, hablante de lengua nheengatu o geral.

En estas comunidades, trabajamos principalmente con cuatro informantes, dos hombres y dos mujeres, AIs bilingües, que eran los principales traductores. La observación participante consistió en el seguimiento de las tareas y vida diaria de los AIS y otros profesionales de la salud, en actividades colectivas y conversaciones con familias apoyadas por los agentes durante un episodio de enfermedad. El registro de observaciones, impresiones, ideas y comentarios en el diario de campo fue amplio y detallado.

Las informaciones recolectadas, que se presentan a continuación, se organizaron para describir y analizar la actuación de los AIs. Buscamos destacar las relaciones que establecen con las formas de atención utilizadas por las familias en la búsqueda de una solución a sus problemas de salud. Dado que las dos comunidades poseen características distintas, optamos por presentar los resultados por separado. Utilizamos seudónimos para preservar la identidad de los interlocutores.

\section{Resultados}

\section{COMUNIDAD BURITI}

La comunidad Buriti se ubica cerca de la desembocadura del Rio Içana, a unas cuatro horas en barco de la ciudad de São Gabriel da Cachoeira. No cuenta con puesto o unidad de salud, pero sí con un AIS. Luis comenzó trabajando como voluntario y hace seis años fue contratado por el DSEI de Alto Rio Negro.

Según relató, sus tareas consistían en atender a las familias enfermas y hacer visitas domiciliarias. Sin embargo, por la falta de suministro de medicamentos por parte del DSEI, su trabajo se veía muy afectado. En ese periodo, su rutina consistía en esperar solicitudes de atención de las familias.
Los momentos de alimentación comunitaria, denominados caribé, ${ }^{5}$ ocurrían por la mañana y al final de la tarde. Eran muy importantes para las actividades de Luis, pues era cuando se enteraba si había alguien enfermo. Durante el caribé, Luis aprovechaba para informar sobre sus actividades, distribuir medicamentos, tomar la presión arterial (PA) de los hipertensos y ofrecer charlas.

Durante el periodo de campo, las actividades desarrolladas por Luis fueron: remoción de pacientes para la sede del DSEI, comunicación con la sede del DSEI por radio, distribución de medicamentos, atención a pacientes, medición de PA de los hipertensos, llenado de fichas de nacidos vivos y recolección de material para la prueba de malaria. Estas actividades formaban parte de la oferta de atención biomédica y no eran supervisadas por ningún profesional de la salud.

La transferencia de las personas enfermas y sus familias a un polo base o a la ciudad, denominada remoción, era una actividad que Luis desempeñaba con frecuencia. La remoción ocurría cuando no había mejoría con los remedios disponibles en la comunidad —alopáticos, caseros o plantas-, cuando no había ningún remedio disponible para atender la situación o cuando el AIs evaluaba a la persona enferma y confirmaba que su estado de salud era grave.

Las remociones eran bastante discutidas con las familias. A veces los AIS afirmaban que era necesario convencerlas para que aceptaran trasladarse a São Gabriel da Cachoeira. ${ }^{6}$ Consideramos que el principal temor en relación con el desplazamiento a

En la región del río Içana, muchas comunidades hacen dos comidas comunitarias, complementarias a lo que se consume en las viviendas. En la comunidad Buriti, el caribé ocurría casi todos los días por la mañana y era menos frecuente al final de la tarde.

6 En otro texto discutimos los dilemas relacionados con las remociones (Pontes, Garnelo y Rego, 2014). 
la ciudad es la pérdida de autonomía que las familias indígenas experimentan cuando están en la Casai.

Las principales quejas de la comunidad respecto al trabajo del AIs Luis eran la escasez de medicamentos, las dificultades que enfrentaba para transferir a las familias al centro urbano y la falta de visitas domiciliarias. Las visitas domiciliarias no tenían orientaciones específicas por parte del DSEI. Según entendían los agentes y las familias indígenas, servían para identificar a las personas enfermas y acompañar a los enfermos en tratamiento.

Al hablar con Luis sobre el comportamiento de las personas cuando estaban enfermas, decía que, en general, las familias se trataban primero en casa, y si no había mejoría, lo buscaban. No obstante, en los primeros días de convivencia, Luis mencionó un segundo agente en la comunidad, el señor Humberto, que trataba a los pacientes con plantas. Luis hizo hincapié en que las familias elegían si lo buscaban primero a él o al señor Humberto. Algunas veces, las familias acudían con Humberto, y si no había mejoría, iban con Luis, pero también podía ocurrir lo contrario. No había diálogo o recomendación de Luis para Humberto, o viceversa. Luis dijo que él también sugería el uso de remedios tradicionales en casos de picadura de serpiente y diarrea, pero conocía pocos, por eso consideraba importante el trabajo de Humberto.

En estos relatos fue evidente que la organización de la atención se estructuraba a partir de la autoatención, o sea, era coordinada por los grupos familiares. La atención ofrecida por Luis o Humberto era evaluada por las familias, y podían darle continuidad o no. En general, el AIs procuraba ofrecer remedios alopáticos para auxiliar un tratamiento comenzado en el ámbito doméstico, y a menudo, también con curadores locales. Incluso cuando las familias consideraban que estaban lidiando con una enfermedad tradicional, solicitaban al agente medicamentos alopáticos.

\section{EL SEGUNDO AGENTE DE LA COMUNIDAD BURITI}

El señor Humberto tenía 65 años de edad, era creyente y había nacido en una comunidad del Alto Içana. Relató que tuvo una enfermedad grave cuando tenía cerca de 30 años de edad y que se trató en la ciudad. Como no mejoraba, comenzó a usar plantas para su tratamiento. Afirmó que aprendió solo, pero que los padres y abuelos le dieron muchos conocimientos al respecto. Después de ese episodio, continuó aprendiendo y comenzó a tratar a otras personas. Humberto narró que recibía visitas de personas de varias comunidades que buscaban su ayuda y pensaba que el DSEI debía contratarlo o pagarle para enseñar a los agentes. Consideraba que su labor era una ayuda, pero podía hacerlo como trabajo.

Humberto identificaba enfermedades de blanco y tradicionales. ${ }^{7}$ Él podía tratar ambas, pero los blancos sólo trataban sus propias enfermedades. Contó que iba al hospital y a la Casai en São Gabriel da Cachoeira con algunos remedios en el bolsillo y trataba a la gente incluso sin que los profesionales supieran. En su percepción, era un doctor indígena. Así se distinguía de los AIs.

Consideraba que los blancos no reconocían su trabajo: "el blanco trabaja con máquinas, es técnico, el indígena no tiene lápices, pero es técnico también [...], uno sólo con los ojos sabe lo que la persona tiene y sabe lo que va a suceder con la persona, porque conoce la enfermedad" (entrevista con Humberto, comunidad Buriti, Alto Rio Negro, 27 de octubre de 2011).

Él creía que debía haber un espacio para que doctores, enfermeros y conocedores locales trabajaran juntos con las dos medicinas. Reforzaba: "si los

\footnotetext{
$7 \quad$ No profundizaremos en las taxonomías de las enfermedades tradicionales en este trabajo. Al respecto, sugerimos la lectura de Garnelo y Buchillet (2006).
} 
blancos quieren aprender de los indígenas, pueden experimentar, y si funciona, pueden trabajar juntos" (entrevista con Humberto, comunidad Buriti, Alto Rio Negro, 27 de octubre de 2011). En su opinión, el paciente debía pasar primero con el conocedor local, experimentar el remedio tradicional, y si no mejorara o no hubiera remedio para su enfermedad, podría buscar tratamiento con los blancos.

\section{COMUNIDAD AÇAÍ}

La comunidad Açaí es la sede de una misión salesiana desde 1952 y de una escuela de nivel fundamental y medio. Para los parámetros regionales, es una localidad con un conjunto poblacional y organizacional diferenciado. La misión salesiana construyó y organizó un ambulatorio con lechos para internación, maternidad, sala para pequeñas cirugías, farmacia y sala de radio. Este ambulatorio formaba parte de la red de la Secretaría Municipal de Salud de São Gabriel da Cachoeira y atendía a residentes de varias comunidades del río Içana. Trabajaban en ese ambulatorio, de lunes a sábado, una médica salesiana, dos técnicas de enfermería y una microscopista. El ambulatorio atendía por demanda espontánea y todas las consultas terminaban con prescripciones medicamentosas múltiples. Muchos pacientes eran personas de la comunidad o que llegaban desplazándose por el río Içana, pero había algunos que frecuentaban el ambulatorio de manera regular.

También trabajaban en Açaí tres AIs: Elena y Rosa, contratadas por el DSEI de Alto Rio Negro, y Bruno, un voluntario. Cada una acompañaba a

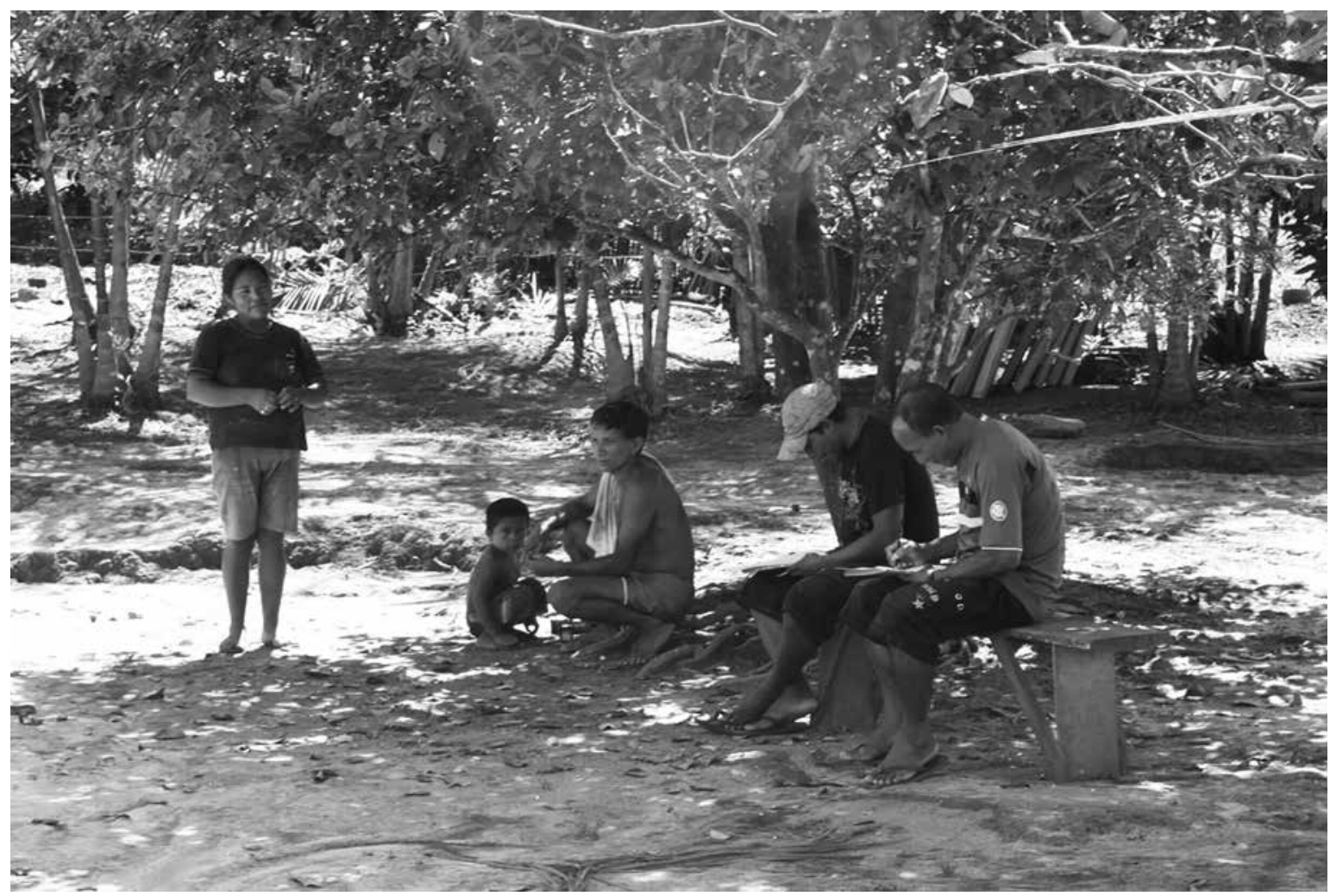

ANA LÚCIA PONTES - Agente indígena de salud entrevista a la población. 
familias y era responsable de un sector diferente. Rosa y Elena decían que a muchas personas no les gustaba la atención ofrecida en el ambulatorio y que preferían que ellas comunicaran sus malestares a la médica y retiraran sus medicamentos. La médica no parecía discrepar de esa manera indirecta de hacer las consultas, que en general eran conversaciones muy rápidas sobre los pacientes y siempre terminaban con la distribución de algún medicamento. En esta relación entre la médica del ambulatorio y la población indígena parecía configurarse una cierta crisis de legitimidad de la medicina científica, como ha sido caracterizada por Ayres (2009), como consecuencia de la pérdida de los aspectos humanistas de la atención a la salud.

Durante el periodo de trabajo de campo, Rosa hizo visitas domiciliarias regulares, tanto en Açaí como en sitios cercanos. Mientras realizaba las visitas, comentaba los problemas de salud de las familias. En sus narraciones siempre mezclaba explicaciones nativas y diagnósticos médicos con la utilización de medicamentos tradicionales e intervenciones biomédicas. Al llegar a los domicilios, hablaba de aspectos de la vida cotidiana y comunitaria, preguntaba si estaban todos bien. Si era necesario, sugería buscar atención médica en el ambulatorio; si había un síntoma que aquejaba a las personas, les distribuía un medicamento.

Elena no hacía visitas domiciliarias con regularidad. Sus revisiones eran más rápidas que las de Rosa, con menos conversación social y dirigidas a preguntar si había alguien enfermo. Decía que muchas personas no le contaban cuando se enfermaban y que las familias se trataban primero con un medicamento tradicional y sólo después la buscaban.

Bruno llevaba tres años como voluntario. Sus visitas también eran cortas y enfocadas a verificar si había enfermos. Cuando los había, Bruno recomendaba primero el uso del remedio casero y si no había mejoría, intentaba algún remedio alopático. A diferencia de las agentes, Bruno era conocedor de plantas y rezos, y era buscado para hacer ese tipo de tratamiento. Mencionaba que las personas que trataba no solían utilizar el ambulatorio como primera opción de atención.

A diferencia de la comunidad Buriti, en Açaí no existía un único conocedor local de referencia. A lo largo del tiempo, se mencionaron algunas personas que conocían las enfermedades y tratamientos tradicionales, el uso de plantas, rezos y bendiciones. En general, las familias tomaban remedios caseros. De acuerdo con la gravedad o persistencia de los síntomas, empezaban un tratamiento tradicional con algún conocedor local o se desplazaban para consultar alguno de su preferencia. También accedían a la atención en el ambulatorio, de manera directa o indirecta, por medio de los agentes.

El ambulatorio, la red del DSEI y la actuación de los AIS representaban el mismo tipo de recurso, es decir, biomédico. La vía para acceder a los servicios de salud eran los AIS o el ambulatorio. Si la enfermedad no tenía solución local o se identificaba la necesidad de recursos disponibles sólo fuera de la comunidad, los agentes contactaban a la sede del DSEI por radio y se procedía a la remoción de las familias.

La discusión sobre los recursos para remoción -bote, motor y gasolina - o su escasez y negociación era un asunto frecuente en las conversaciones en el ambulatorio y la sala de radiocomunicación. Las familias también relataron experiencias negativas en la Casai y en los servicios de salud urbanos, por ello consideraban que había que posponer la remoción el mayor tiempo posible.

Cabe mencionar que algunas de las personas que eran atendidas en el ambulatorio se desplazaban por el Rio Içana y aprovechaban para frecuentarlo. En un área donde la disponibilidad de recursos es restringida, cualquier oportunidad de acceso a ellos, en cantidad o diversidad, es muy valorada en todos los ámbitos de la vida, incluso en la salud. 


\section{Discusión}

La diversidad de formas de atención en las dos comunidades estudiadas involucra la biomedicina, la medicina tradicional y la autoatención. Destacamos que en los territorios indígenas encontramos contextos de pluralidad médica (Menéndez, 2009). Esas formas de atención se articulan a partir de las elecciones de las familias, sus experiencias previas y la efectividad de cada forma de atención ante sus necesidades (Menéndez, 2009). La complementariedad entre formas de atención se establece por medio de las prácticas de autoatención de los sujetos y grupos familiares, y no de los servicios de salud, como usualmente sugieren las políticas públicas.

En la comunidad Buriti, se accede a la atención biomédica por medio del AIs, lo que implica la disponibilidad de algunos medicamentos para aliviar los síntomas o la remoción de personas y familias a los servicios de salud en el centro urbano. En la comunidad Açaí era posible acceder a la atención médica y de enfermería de manera directa en el ambulatorio, pero había familias que preferían que ese contacto fuera mediado por los AIS.

En nuestro análisis, los AIS no son especialistas o curadores profesionales de la atención biomédica, pues no poseen formación profesional ni reconocimiento social. ${ }^{8}$ Para las familias de las comunidades indígenas del Alto Rio Negro, los agentes son responsables de poner a disposición los recursos de los servicios biomédicos de salud, situación encontrada en otros contextos (Langdon, Diehl y DiasScopel, 2014). Este papel es fundamental, pues los pueblos indígenas deben traspasar varias barreras para acceder a los servicios de salud (Gomes y Esperidião, 2017). En cuanto al acceso entendido como "la capacidad de un grupo para buscar y obtener atención” (Giovanella y Fleury, 1996: 189), los AIS pueden concebirse como facilitadores de ese proceso para las familias indígenas.
A pesar de que su papel es ofrecer y facilitar la atención biomédica, los AIS reconocían también los diagnósticos y tratamientos tradicionales. Como señalan Scopel, Dias-Scopel y Langdon (2015), los AIS en las comunidades estudiadas tenían pleno conocimiento de las prácticas de autoatención de las familias. Las narrativas y los itinerarios terapéuticos de las familias indígenas referían términos y terapias de ambos sistemas médicos, lo que muestra que mezclaban, articulaban y complementaban sus tratamientos.

En el ámbito de la atención biomédica, se destacó la importancia atribuida por los AIs y las familias al acceso a los medicamentos alopáticos. Concordamos con Scopel, Dias-Scopel y Langdon (2015) en que la forma de acción de los AIS en la formulación de diagnósticos e indicación de uso de medicamentos se asemeja a los procesos de autoatención de las familias, por lo que puede caracterizarse como una práctica de automedicación. Según Menéndez, "la automedicación, como parte de la autoatención, quiere abatir, limitar, dar solución provisoria, en forma definitiva o transitoria a los padecimientos, daños, problemas" (1992: 155). La prescripción de la medicación en ese ámbito se hace con base en indicadores diagnósticos (Menéndez, 2009), manejados tanto por las familias como por los AIS, y hay coincidencias entre ellos, tanto en la medicina científica y tradicional, como fiebre, tos y diarrea.

En coherencia con la tendencia general de la biomedicina de criticar y reprimir las prácticas de automedicación (Menéndez, 1992), en los últimos

8

En el DSEl de Alto Rio Negro, los AIs no reciben ningún tipo de formación inicial o material informativo sobre su trabajo, son orientados por los enfermeros de sus equipos, principalmente por Als más antiguos. La mayoría de los agentes del DSEI de Alto Rio Negro estaban matriculados en el Curso Técnico de Agentes Comunitarios Indígenas de Salud, en el cual participaba una de las investigadoras. 


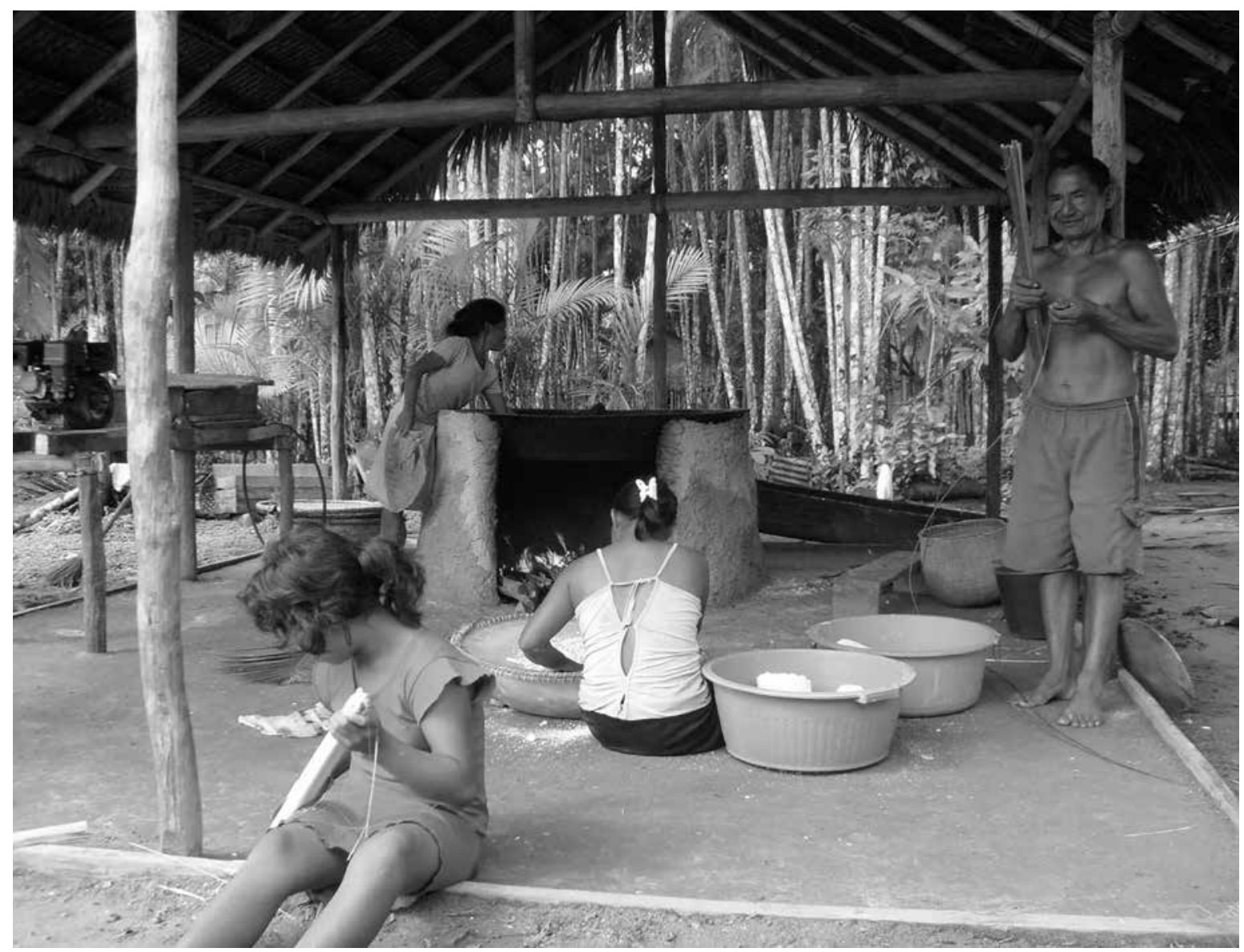

ANA LÚCIA PONTES • Elaboración de casabe.

años existe una recomendación explícita para la disminución del acceso de los AIS a los medicamentos. En el entendimiento de los gestores y los profesionales del DSEI, la prescripción forma parte del ámbito exclusivo de la práctica médica.

Estas recomendaciones ignoran que los servicios y los profesionales de la salud estimulan las prácticas de automedicación (Menéndez, 1992). Además, en el contexto indígena, incluso en el Alto Rio Negro, la consolidación del papel de los AIS desde la década de 1980 se relacionó con su entrenamiento para la prescripción y distribución de medicamentos, hasta antibióticos. Hasta el momento, gestores y profesionales todavía refuerzan que el trabajo del AIS es identificar y tratar a los enfermos, que no tienen otra función, salvo la distribución de algunos medicamentos.

Si consideramos que la automedicación es impulsada por la expansión de la biomedicina, aliada tanto de la industria farmacéutica como de los aparatos del Estado, nos preguntamos si los AIS forman parte de los procesos de medicalización en territorios indígenas. ${ }^{9}$ Foucault caracteriza la

$9 \quad$ Pese a las limitaciones señaladas por Menéndez (1992) sobre la unilateralidad de algunos estudios de los procesos de medicalización, consideramos que ésta no produce contradicciones en nuestro análisis. 
medicalización como el proceso en el que la medicina pasa a controlar el comportamiento, la conducta y el cuerpo humano:

El control de la sociedad sobre los individuos no se opera simplemente por la consciencia o por la ideología, sino que se ejerce en el cuerpo, con el cuerpo. Para la sociedad capitalista lo importante era lo biológico, lo somático, lo corporal antes que nada. El cuerpo es una realidad biopolítica; la medicina es una estrategia biopolítica (1977: 5).

La expansión de la medicalización ocurre con la constitución de la medicina de Estado, a partir del siglo XVIII, y coincide con la emergencia de la medicina científica. Foucault (1977) destaca su carácter biologizante. La medicina estatal se conforma en un proceso de normalización de la práctica y el saber médicos, que después se aplica a la atención de los pacientes; el establecimiento de una organización administrativa que pasa a controlar la actividad de los médicos, y por último, por la creación de cargos de médicos de gobierno que asumen la responsabilidad y poseen autoridad sobre la salud de la población de un territorio. Así, se establece una medicina "burocratizada, colectivizada y estatizada” (1977: 10).

El debate de la medicalización tiene relevancia, pues demuestra el continuo proceso de conversión de fenómenos de la vida y situaciones cotidianas en problemas médicos o enfermedades, que serán susceptibles de control y tratamiento medicamentoso o quirúrgico. Además de la medicina de Estado, la biologización es otro elemento estructurante de la biomedicina y la medicalización que consiste en el fundamento en las investigaciones biomédicas para el entendimiento de la causalidad de las enfermedades y sus soluciones farmacológicas (Menéndez, 2009). La expansión del consumo de medicamentos es uno de los marcadores de la expansión de la biomedicina y de su relación con la expansión capitalista, incluso en lugares remotos, como la región del Alto Rio Negro.
En el contexto de las comunidades indígenas en el Alto Rio Negro, podemos señalar que la estructura médica del Estado garantiza su acción continua en los territorios indígenas con la inserción de los AIS en los equipos de salud. En el DSEI de Alto Rio Negro, los profesionales de los equipos tienen alta rotación y visitan las comunidades de manera irregular, ${ }^{10}$ situación encontrada por otros investigadores (Langdon, Diehl y Dias-Scopel, 2014).

Por otro lado, la medicalización identificada en el trabajo de los AIS se relaciona con la prescripción y distribución de medicamentos y no con la biologización del curso de salud/enfermedad/atención. La actuación de los AIs en ese curso se asemeja a las prácticas de autoatención, de modo que sugerimos que se entienda como automedicación. Con ello pretendemos destacar que el desarrollo de la medicalización está incluido "en un proceso previo y permanente de autoatención a la enfermedad" (Menéndez, 1992: 155). Sobresale, según lo señalado por Fóller (2004), que en la evolución de la incorporación de los medicamentos, los indígenas poseen agencia social y producen nuevos discursos y prácticas: "la autoatención supone un proceso de síntesis [y] la potencialidad de inclusión de nuevas técnicas, productos y creencias que se integran al saber existente [...] y ello favorece [su continua] transformación" (Menéndez, 1993: 74).

Otro aspecto - en el que concordamos con Guimarães, quien lo identificó en el contexto sanumá- es que para los indígenas "las terapias biomédicas son vistas como acciones que se centran en síntomas y sensaciones desagradables inmediatas" (2015: 2154), lo que no interfiere con las dimensiones causales de las enfermedades —epistemológicas - que son tratadas por las medicinas

10 En el periodo de trabajo de campo, los equipos no visitaban las comunidades desde hacía seis meses por problemas de renovación de contratos. 
tradicionales. Si consideramos la vulnerabilidad de esas poblaciones, la automedicación funciona como una estrategia de aumento de la oferta de recursos de salud y forma parte de las prácticas de autoatención de las familias indígenas.

Los indígenas de la región no rechazan la biomedicina; de hecho, identifican que da respuestas satisfactorias a los problemas de salud que emergieron del contacto con el blanco. No obstante, hacen diversas críticas al modelo biomédico ejecutado por el DSEI, por su baja efectividad y por una insatisfacción con los tratamientos proporcionados a los indígenas en los servicios. En el caso de los profesionales de la salud, se trata de una "superioridad tecnológica ilusoria y de una capacidad de protección irreal” (Souza Lima, 2014: 52-53). Además, los indígenas relatan vivencias negativas en los servicios de salud debido a violencias y formas de discriminación, ${ }^{11}$ que generan un deseo de "aproximación controlada" a los servicios de salud. En este sentido, la autoatención aparece como una estrategia en el mantenimiento de la autonomía de los modos indígenas de lidiar con cuestiones relativas al proceso salud/enfermedad/atención y de la articulación a la biomedicina.

Identificamos en las comunidades estudiadas las categorías emic de enfermedad y remedio de blanco y tradicional. La medicina y los remedios del blanco se refieren a aquellos que surgieron después del contacto interétnico y los identificamos con la atención biomédica. Las enfermedades y la medicina tradicional se apoyan en un sistema de saberes míticos y en las formas de organización de parentesco (Garnelo y Wright, 2001; Garnelo y Buchillet, 2006). Destacamos la clasificación de Garnelo y Buchillet (2006) sobre las enfermedades tradicionales baniwa, que incluyen padecimientos relacionados con la agresión de seres-espíritus; envenenamientos — una forma de brujería que tiene un lugar central en la cosmología baniwa-; conflictos de género, que remiten a las guerras ancestrales entre hombres y mujeres; enfermedades provocadas por yoópinai, seres-espíritus del bosque, y enfermedades producto del contacto, que se han insertado en la cosmología tradicional. De la misma forma, "la curación remite a la instauración de estrategias de resolución de conflictos que amenazan el orden cósmico y social, fundado en la jerarquía e intercambio de bienes y servicios entre consanguíneos y afines, jóvenes y viejos, hombres y mujeres" (2006: 233).

Los conflictos y asimetrías existentes en las relaciones intermédicas (Fóller, 2004), entre medicina del blanco y tradicional, se pusieron en evidencia en los discursos del segundo agente, el señor Humberto, quien se refirió a la falta de reconocimiento por parte del blanco de la eficacia de la medicina tradicional. En su argumentación, para defender la equivalencia entre ambas medicinas, hace uso de terminologías y criterios del propio discurso hegemónico, al igual que de los aspectos "técnicos" de su trabajo. Vemos en el discurso de ese curador los efectos hegemónicos de la biomedicina, en cuanto a la devaluación del conocimiento tradicional en la zona de contacto entre biomedicina y pueblos indígenas (Follér, 2004).

\section{Consideraciones finales}

Las prácticas de autoatención de las familias indígenas fueron la primera instancia de formulación de diagnóstico y resolución de los problemas de salud

Los indígenas comentaban que tenían miedo de ser transferidos para ser tratados en la ciudad, pues se les prohíbe utilizar sus prácticas tradicionales de cura dentro de los servicios de salud, notan que sus dietas y restricciones alimentarias no son respetadas - reciben comidas inadecuadas-, son obligados a convivir con grupos de "enemigos", los alojamientos son precarios y con frecuencia sus decisiones no son escuchadas. Todas estas situaciones representan vivencias negativas y violentas para ellos. 


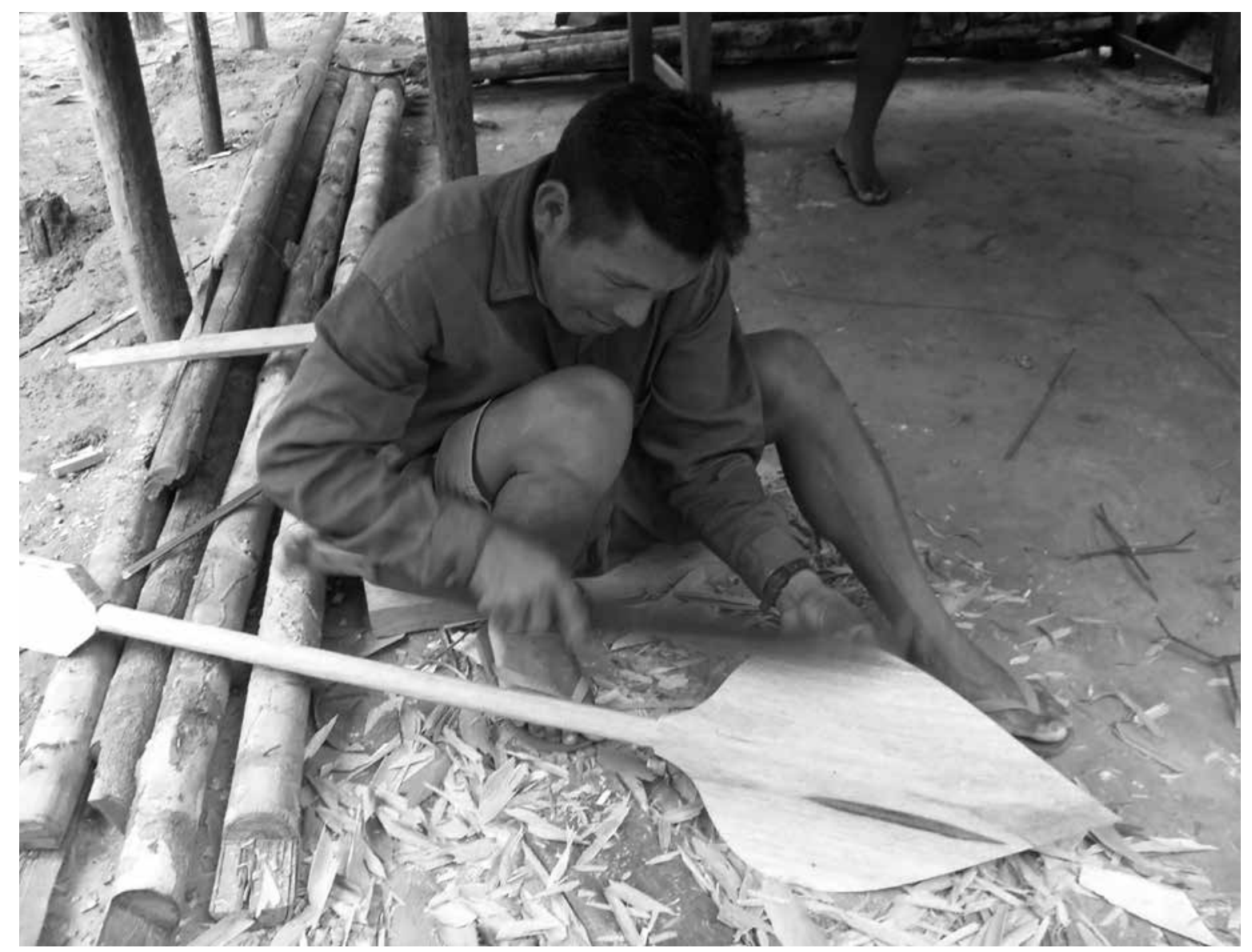

ANA LÚCIA PONTES • Fabricación de remos en Rio Negro.

en las comunidades estudiadas, asunto que también ha sido identificado en otros contextos indígenas (Yajahuanca, Diniz y Cabral, 2015; Scopel, DiasScopel y Langdon, 2015). Así, a diferencia de lo que preconizan los marcos del modelo de organización de los DSEI (Fundação Nacional de Saúde, 2002), el primer nivel de atención del sistema sería la autoatención. Menéndez (2009) propone que la autoatención debería ser el eje de la organización de los sistemas de salud locales y Langdon (2004) considera que la atención diferenciada es mejor entendida a partir de esa articulación. En ese sentido, destacamos el pluralismo médico existente en los territorios indígenas.
La autoatención como primer nivel de atención de los sistemas de salud indígenas es resolutiva para muchos problemas que enfrentan los indígenas y su saber se transforma a partir del contacto con otros saberes, incluso con el biomédico. La actuación de los AIs facilita el acceso a los servicios biomédicos y consideramos que su trabajo funciona como un generador de prácticas de autoatención relacionadas con el uso de medicamentos en un proceso similar a la automedicación. Este dato también muestra la centralidad de los medicamentos en las relaciones transaccionales entre autoatención y biomedicina, según lo señalado por Menéndez (2009). Estamos de acuerdo con Fóller cuando destaca que en los 
procesos de resistencia de los pueblos indígenas en el contacto intermédico "este medio término implica tanto aceptar el conocimiento biomédico como resistir al poder y a la ideología de la biomedicina"
(2004: 144). Concluimos que las prácticas de autoatención, incluso la automedicación, son estrategias de resistencia y de mantenimiento de la autonomía de los pueblos indígenas. D

\section{Bibliografía}

Andrello, Geraldo, 2006, Cidade do indio: transformações e cotidiano em lauaretê, Editora Universidade Estadual Paulista/Instituto Socioambiental/Núcleo de Transformações Indígenas, São Paulo y Río de Janeiro.

Ayres, José Ricardo C. M., 2009, "Cuidado e reconstrução das práticas de saúde”, en José Ricardo C. M. Ayres, Cuidado: trabalho e interação nas práticas de saúde, Centro de Estudos e Pesquisa em Saúde Coletiva/Universidade do Estado do Rio de Janeiro-Instituto de Medicina Social/Associação Brasileira de Saúde Coletiva, Río de Janeiro, pp. 41-74.

Basta, Paulo Cesar, Jesem Douglas Yamall Orellana y Rui Arantes, 2012, "Perfil epidemiológico dos povos indígenas no Brasil: notas sobre agravos seleccionados”, en Luiza Garnelo y Ana Lúcia Pontes (orgs.), Saúde indígena: uma introdução ao tema, Ministério da EducaçãoSecretaria de Educação Continuada, Alfabetização, Diversidade e Inclusão, Organización de las Naciones Unidas para la Educación, la Ciencia y la Cultura, Brasilia, pp. 60-106.

Cardoso, Marina, 2015, "Políticas de saúde indígena no Brasil: do modelo assistencial à representação política", en Esther Jean Langdon y Marina Cardoso (orgs.), Saúde indigena: políticas comparadas na América Latina, Universidade Federal de Santa Catarina, Florianópolis, pp. 83-106.

Chizzotti, Antonio, 2010, Pesquisa qualitativa em ciências humanas e sociais, Vozes, Petrópolis.

Erthal, Regina M. de Carvalho, 2003, "A formação do agente de saúde indígena Tikúna no Alto Solimões: uma avaliação crítica”, en Carlos E. A. Coimbra Jr., Ricardo Ventura Santos y Ana Lúcia Escobar (orgs.), Epidemiologia e saúde dos povos indígenas no Brasil, Fundação Oswaldo Cruz, Río de Janeiro, pp. 197-216.

Ferreira, Luciane Ouriques, 2013, Medicinas indigenas e as políticas da tradição: entre discursos oficiais e vozes indígenas, Fundação Oswaldo Cruz, Río de Janeiro.

__ , 2015, "Interculturalidade e saúde indígena no contexto das políticas públicas brasileiras", en Esther Jean Langdon y Marina Cardoso (orgs.), Saúde indígena: políticas comparadas na América Latina, Universidade Federal de Santa Catarina, Florianópolis, pp. 217-246.

Follér, Maj-Lis, 2004, "Intermedicalidade: a zona de contato criada por povos indígenas e profissionais de saúde”, en Esther Jean Langdon y Luiza Garnelo (orgs.), Saúde dos povos indígenas: reflexões sobre antropologia participativa, Associação Brasileira de Antropologia/ ContraCapa, Río de Janeiro, pp. 129-148.

Foucault, Michel, 1977, "Historia de la medicalización”, en Educación Médica y Salud, vol. 11, núm. 1, pp. 3-25.

Fundação Nacional de Saúde, 2002, Política nacional de atenção à saúde dos povos indígenas, Ministério da Saúde, Brasilia.

Garnelo, Luiza, 2003, Poder, hierarquia e reciprocidade: saúde e harmonia entre os Baniwa do Alto Rio Negro, Fundação Oswaldo Cruz, Río de Janeiro.

2012, "Política de Saúde Indígena no Brasil: notas sobre as tendências atuais do processo de implantação do subsistema de atenção á saúde", en Luiza Garnelo y Ana Lúcia Pontes (orgs.), Saúde indígena: uma introdução ao tema, Ministério da Educação-Secretaria de Educação Continuada, Alfabetização, Diversidade e Inclusão/Organización de las Naciones Unidas para la Educación, la Ciencia y la Cultura, Brasilia, pp. 18-59.

__ 2014, "O sus e a saúde indígena: matrizes políticas e institucionais do Subsistema de Saúde Indígena", en Carla Costa Texeira (org.), Saúde indígena em perspectiva: explorando suas matrizes históricas e ideológicas, Fundação Oswaldo Cruz, Río de Janeiro, pp. 107-144.

Garnelo, Luiza y Dominique Buchillet, 2006, "Taxonomias das doenças entre os índios baniwa (arawak) e desana (tukano oriental) do Alto Rio Negro (Brasil)”, en Horizontes Antropológicos, vol. 12, núm. 26, pp. 231-260.

Garnelo, Luiza y Robin Wrigth, 2001, "Doença, cura e serviços de saúde. Representações, práticas e demandas Baníwa”, en Cadernos de Saúde Pública, vol. 17, núm. 2, pp. 273-284. 
Giovanella, Ligia y Sonia Fleury, 1996, “Universalidade da atenção à saúde: acesso como categoria de análise”, en Catalina Eibenschutz (org.), Política de saúde: o público e o privado, Fundação Oswaldo Cruz, Río de Janeiro, pp. 177-198.

Gomes, Silvana Cardoso y Monique Azevedo Esperidião, 2017, "Acesso dos usuários indígenas aos serviços de saúde de Cuiabá, Mato Grosso, Brasil", en Cadernos de Saúde Pública, vol. 33, núm. 5, pp. 1-20.

Guimarães, Sílvia Maria Ferreira, 2015, “El sistema médico sanumá-yanomami y la atención a la política de salud indígena”, en Cadernos de Saúde Pública, vol. 31, núm. 10, pp. 2148-2156.

Instituto Brasileiro de Geografia e Estatística (IBGE), 2012, Os indígenas no Censo Demográfico 2010: primeiras considerações com base no quesito cor ou raça, Instituto Brasileiro de Geografia e Estatística, Río de Janeiro.

Instituto Socioambiental (ISA), 2017, “Etnias do Rio Negro”, en Povos indígenas no Brasil. Disponible en línea: <https://pib.socioambiental.org/ pt/Povo:Etnias_do_Rio_Negro>. Consultado el 8 de octubre de 2017.

Instituto Socioambiental (ISA) y Federação das Organizações Indígenas do Rio Negro (FoIRN), 2006, Mapa-livro povos indígenas do Rio Negro: uma introdução à diversidade socioambiental do noroeste da Amazônia brasileira, Instituto Socioambiental/Federação das Organizações Indígenas do Rio Negro, São Paulo y São Gabriel da Cachoeira.

Langdon, Esther Jean, 2004, “Uma avaliação crítica da atenção diferenciada e a colaboração entre antropologia e profissionais de saúde”, en Esther Jean Langdon y Luiza Garnelo (orgs.), Saúde dos povos indígenas: reflexões sobre antropologia participativa, Associação Brasileira de Antropologia/ContraCapa, Río de Janeiro, pp. 33-52.

Langdon, Esther Jean, Eliana Elisabeth Diehl y Raquel Paiva Dias-Scopel, 2014, "O papel e a formação dos agentes indígenas de saúde na atenção diferenciada à saúde dos povos indígenas brasileiros”, en Carla Costa Texeira (org.), Saúde indígena em perspectiva: explorando suas matrizes históricas e ideológicas, Fundação Oswaldo Cruz, Río de Janeiro, pp. 213-240.

Menéndez, Eduardo L., 1987, “Medicina tradicional' o sistemas práctico-ideológicos de los conjuntos sociales, como primer nível de atención”, en El futuro de la medicina tradicional en la atención a la salud de los países latinoamericanos, Centro Interamericano de Estudios de Seguridad Social, México, pp. 37-62.

_ 1992, "Autoatención y automedicación. Un sistema de transacciones sociales permanentes", en Roberto Campos Navarro (comp.), La antropología médica en México, Universidad Autónoma Metropolitana, México, pp. 141-185.

—_, 1993, "Autoatención y participación social: estrategias o instrumentos en las políticas de atención primaria”, en Medicina tradicional 500 años después: historia y consecuencias actuales, Instituto de Medicina Dominicana, Santo Domingo, pp. 62-104.

—_ 2003, "Modelos de atención de los padecimientos: de exclusiones teóricas y articulaciones prácticas", en Ciência \& Saúde Coletiva, vol. 8, núm. 1, pp. 185-208.

—_, 2009, De sujetos, saberes y estructuras: introducción al enfoque relacional en el estudio de la salud colectiva, Lugar Editorial, Buenos Aires.

Novo, Marina Pereira, 2009, "Saúde e interculturalidade: a participação dos Agentes Indígenas de Saúde/Alss do Alto Xingu”, en R@U. Revista de Antropologia da UfSCar, vol. 1, núm. 1, pp. 122-147.

Pontes, Ana Lúcia, 2013, Atenção diferenciada e o trabalho do agente indigena de saúde na implementação da política de saúde indígena, tesis de doctorado en salud pública, Escola Nacional de Saúde Pública Sergio Arouca, Río de Janeiro.

Pontes, Ana Lúcia, Luiza Garnelo y Sergio Rego, 2014, "Reflexões sobre questões morais na relação de indígenas com os serviços de saúde", en Revista Bioética, vol. 22, núm. 2, pp. 337-346.

Pontes, Ana Lúcia, Sergio Rego y Luiza Garnelo, 2015, "O modelo de atenção diferenciada nos Distritos Sanitários Especiais Indígenas: reflexões a partir do Alto Rio Negro/AM, Brasil”, en Ciência \& Saúde Coletiva, vol. 20, núm. 10, pp. 3199-3210.

Presidência da República, 1990, "Lei núm. 8.080, de 19 de setembro de 1990. Dispõe sobre as condições para a promoção, proteção e recuperação da saúde, a organização e o funcionamento dos serviços correspondentes e dá outras providências". Disponible en línea: <http://conselho.saude.gov.br/legislacao/lei8080_190990.htm>.

__ 1999, "Lei núm. 9.836 de 23 de setembro de 1999. Acrescenta dispositivos à Lei no 8.080, de 19 de setembro de 1990, que 'dispõe sobre as condições para a promoção, proteção e recuperação da saúde, a organização e o funcionamento dos serviços correspondentes e dá outras providências', instituindo o Subsistema de Atenção à Saúde Indígena”, en Diário Oficial da União, 24 de septiembre.

Scopel, Daniel, Raquel Paiva Dias-Scopel y Esther Jean Langdon, 2015, “Intermedicalidade e protagonismo: atuação dos agentes indígenas de saúde munduruku da Terra Indígena Kwatá-Laranjal, Amazonas, Brasil”, en Cadernos de Saúde Pública, vol. 31, núm. 12, pp. $2559-2568$. 
Silva Junior, Aluisio Gomes da, 2006, Modelos tecnoassistenciais em saúde: o debate no campo da saúde coletiva, Hucitec, São Paulo.

Sistema de Informação da Atenção à Saúde Indígena (SIASI), 2013, "Dados populacionais de 2013 dos indígenas cadastrados no SIASI do DSEI".

Disponible en línea: <http://dw.saude.gov.br/gsid/servlet/mstrWeb?src=mstrWeb.2048001\&evt=2048001\&share=1\&hiddensections= header\%2Cpath\%2CdockLeft\%2Cfooter\&visMode=0\&currentViewMedia=2\&documentID=9655D54A11E35375B9F80080EF857719\&S erver=SRVBIPDF03\&Port=0\&Project=DMSIASI_4\&>. Consultado el 8 de octubre de 2017.

Souza Lima, Antonio Carlos, 2014, "Revisitando a tutela: questões para se pensar as políticas públicas para povos indígenas", en Carla Costa Texeira (org.), Saúde indígena em perspectiva: explorando suas matrizes históricas e ideológicas, Fundação Oswaldo Cruz, Río de Janeiro, pp. 27-58.

Teixeira, Carmen Fontes y Jorge Pereira Solla, 2006, Modelo de atenção à saúde: promoção, vigilância e saúde da família, Editora da Universidade Federal da Bahia, Salvador.

Wright, Robin, 1992, História indigena do noroeste da Amazônia, en Manuela Carneiro da Cunha (org.), Historia dos índios no Brasil, Companhia das Letras/Secretaria Municipal de Cultura/Fundação de Amparo à Pesquisa do Estado de São Paulo, São Paulo, pp. 253-266.

Yajahuanca, Rosário Avellaneda, Carmen Simone Grillo Diniz y Cristiane da Silva Cabral, 2015, “É preciso 'ikarar os kutipados': interculturalidade e assistência à saúde na Amazônia Peruana”, en Ciência \& Saúde Coletiva, vol. 20, núm. 9, pp. 2837-2846.

\section{Entrevistas}

Humberto, comunidad Buriti, Alto Rio Negro, Brasil, 27 de octubre de 2011. 